\title{
Nutrition in Hepato-Biliary Pancreas Surgery
}

\section{Ho-Seong Han}

Department of Surgery,

Seoul National University College of Medicine, Korea

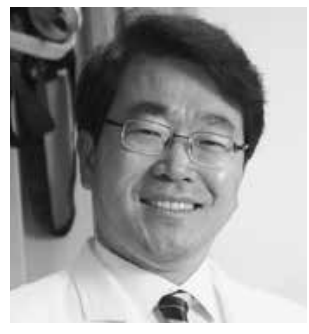

HBP surgery field is special as surgery is usually extensive and complicated. Therefore, nutritional therapy on HBP surgery is also dealt with special attention. Most challenging surgery in HBP field are pancreaticoduodenectomy (PD), major liver resection, and liver transplantation. The above patients are already under nutrition due to cancer, chronic liver disease or cirrhosis. And these diseases are associated with high rates of postoperative morbidity. The benefits of nutritional support are well documented. Especially, in pancreaticoduodenectomy, there also reports that early enteral nutrition are widely accepted. However, fear of postoperative ileus and anastomotic leakage has been the barrier for early feeding after abdominal surgery. Prospective study has been performed to assess the safety, tolerability and outcome of early enteral feeding after PD or other major HBP operations. Liver cirrhotic patient need special treatment form nutritional therapy team. Recently, ERAS is applied for the treatment of patients with HBP disease. This allows more rapid recovery, early discharge, and back to normal life. The activities of NST (nutritional therapy team) may help to reduce the operation related morbidities. Therefore, governmental decision for national coverage for the activities is very necessary. This presentation is focused on various aspects on nutritional treatment for the patients for HBP surgery. 